\title{
Some Properties and Stability Results for Sector-Bounded LTI Systems
}

\author{
Sandeep Gupta \\ ViGYAN, Inc. \\ Hampton, VA 23666 \\ Suresh M. Joshi \\ NASA Langley Research Center \\ Hampton, VA 23681
}

To be presented at

The $33^{\text {rd }}$ IEEE Conference on Decision and Control Lake Buena Vista, FL, December 14-16, 1994. 
. 


\title{
Some Properties and Stability Results for Sector-Bounded LTI Systems
}

\author{
Sandeep Gupta ${ }^{1}$ \\ ViGYAN Inc., Hampton VA 23666 \\ Suresh M. Joshi \\ NASA Langley Research Center, Hampton VA 23681
}

\begin{abstract}
This paper presents necessary and sufficient conditions for a linear, time-invariant (LTI) system to be inside sector $[a, b]$ in terms of linear matrix inequalities in its state-space realization matrices, which represents a generalization of similar conditions for bounded $\mathcal{H}_{\infty}$-norm systems. Further, a weaker definition of LTI systems strictly inside sector $[a, b]$ is proposed, and state-space characterization of such systems is presented. Sector conditions for stability of the negative feedback interconnection of two LTI systems and for stability of LTI systems with feedback nonlinearities are investigated using the Lyapunor function approach. It is shown that the proposed weaker conditions for an LTI system to be strictly inside a sector are sufficient to establish closed-loop stability of these systems.
\end{abstract}

\section{Introduction}

Sector conditions for the stability of the feedback interconnection of general input-output systems were introduced in [1] and further expanded upon in [2,3]. These results were developed in an abstract mathematical setting of general inputoutput maps using operator-theoretic methods. In this paper we first present a state-space characterization of linear, timeinvariant (LTI) systems inside sector $[a, b]$, in terms of three matrix relations in its system matrices, which is referred to as the sector-boundedness lemma, since it represent a generalization of the bounded realness lemma for bounded $\mathcal{H}_{\infty}$-norm systems [4,5]. Moreover, this state-space characterization of sectorbounded LTI systems can be equivalently expressed in terms of linear matrix inequalities, as shown in Theorem 1. Using these state-space characterizations, analysis of sector bounds on stable LTI systems can be performed with algebraic Riccati equations as well as convex programming techniques for the solution of linear matrix inequalities [6]. Next, a new definition is proposed for LTI systems strictly inside a sector, which is weaker than the corresponding definition in previous literature [1,2], and extends the notion of strict bounded realness $[4,5]$ as well as strict positive realness [7]. State-space characterization of LTI systems strictly inside a sector is presented in Theorem 2 . The next result

\footnotetext{
${ }^{1}$ Member IFEE, assigned to Guidance and Control Branch, Mail Stop 161, Fight Dynamics and Control Division. NASA Langley Research Cenler, Hampton, VA.

${ }^{2}$ Fellow IEEE, Dynamics and Controls Branch, Mail Stop 489, Fight Dynamics and Control Division. NASA Langley Research Center, Hampton, VA.
}

in Theorem 3 demonstrates that the weaker definition of systems strictly inside a sector is adequate to establish sector conditions for closed-loop stability of the negative feedback interconnection of two LTI systems [1,2], thus extending the previously available results. Theorem 4 presents a similar extension for sector conditions to guarantee stability of LTI systems with feedback nonlinearities. Moreover, these results are established using straightforward Lyapunov function techniques rather than the abstruse operator theoretic methods. Sector conditions for closedloop stability are seen to be a generalization of the small gain theorem, and the ensuing discussion shows that these conditions may be used for synthesis of less conservative robust controllers. as opposed to the small gain theorem-based controllers.

Before proceeding, we present the notation used in the developments of this paper. Herm $\{M\}$ is the Hermitian part of matrix $M$, that is, $\operatorname{Herm}\{M\}=\frac{1}{2}\left(M+M^{*}\right)$. where the superscript "denotes the conjugate transpose of the matrix; and $M^{T}$ is the transpose of the matrix, $M$. A stable linear time-invariant system, $\Sigma$, is represented in state-space form as $\dot{x}=A x+B u, \quad y=C x+D u$, where $x \in \mathbf{R}^{n}$, $u \in \mathbf{R}^{m}$ and $y \in \mathbf{R}^{m}$, with $n \geq m$. The square transfer function matrix of $\Sigma$, is $G(s)=C(s I-A)^{-1} B+D$, and the quadruple $[A, B, C, D]$ is a state-space representation of $G(s) . G^{\sim}(s) \triangleq G^{T}(-s)=B^{T}\left(-s I-A^{T}\right)^{-1} C^{T}+D^{T}$; thus, the quadruple $\left[-A^{T},-C^{T}, B^{T}, D^{T}\right]$ is a state-space representation of $G^{\sim}(s)$. A square matrix transfer function, $\phi(s)$ is said to be parahermitian if $\phi^{\sim}(s)=\phi(s)$. The next section discusses the conditions for sector boundedness of LTI systems.

\section{Sector-Bounded LTI Systems}

A memoryless, time-varying nonlinearity, $\psi(y, t)$, is said to be inside sector $[a, b]$ if $(\psi-b y)^{T}(\psi-a y) \leq 0$ for all $y \in \mathbf{R}^{m}$ [1]. Geometrically, these sector conditions imply that the graph of the nonlinearity lies within a conical region in the $\mathbf{R}^{m} \times \mathbf{R}^{m}$ input-output space for all time, $t$. For $m=1$, Figure 1 shows a nonlinearity, $\psi(y, t)$, inside sector $[a, b]$; the graph of $\psi(y, t)$ must lie in the shaded region within two lines of slopes $a$ and $b$. The concept of sector-boundedness extends to linear time-invariant systems by defining an LTI system, $\Sigma$, to be inside sector $[a, b]$ if $\operatorname{Herm}\left\{[G(j \omega)-a I]^{*}[G(j \omega)-b I]\right\} \leq 0$, for all $\omega \in \boldsymbol{R}[10]$. The Nyquist plot of an LTI system, inside a sector $[a, b], b>a$, lies within a circle in the frequency plane, whose 
center is at $[(a+b) / 2, j 0]$ and has a radius of $(b-a) / 2$. Note that a square, bounded, real system, that is, a system satisfying $\|G(s)\|_{\infty} \leq 1$, is inside sector $[-1,1]$; and its Nyquist plot lies within a unit circle centered at the origin. For example, $G(s)=15 /(s+3)(s+5)$ is inside sector $[-0.4,1.0]$ and its Nyquist plot lies within the corresponding circle, shown in Figure 2. Note that $\|G(s)\|_{\infty} \leq 1$, so that its Nyquist plot also lies within the unit circle centered at the origin, as shown in Figure 2.

The bounded realness lemma [4,5] gives a state-space characterization of $\mathcal{H}_{\infty}$-norm bounded LTI systems; and these conditions may equivalently be expressed as linear matrix inequalities [11]. Theorem 1 below presents the extension of these state-space characterizations to LTI systems inside sector $[a, b]$.

Theorem 1: Given a stable LTI system $\Sigma: \quad \dot{x}=A x+$ $B u, y=C x+D u$, where the quadruple $[A, B, C, D]$ is a minimal realization of the transfer function matrix, $G(s)=$ $C(s I-A)^{-1} B+D$, the following statements are equivalent.

(i) $\Sigma$ is inside sector $[a, b]$.

(ii) There exist real matrices $P=P^{T}>0, L$ and $W$ which satisfy

$$
\begin{aligned}
& P A+A^{T} P=-C^{T} C-L^{T} L \\
& P B=C^{T}(\alpha I-D)-L^{T} W \\
& D^{T} D-\alpha\left(D+D^{T}\right)+a b I=-W^{T} W
\end{aligned}
$$

where $\alpha=(a+b) / 2$.

(iii) There exists a real matrix $P=P^{T}>0$ which satisfies the linear matrix inequality

$$
\left[\begin{array}{cc}
C^{T} C+P A+A^{T} P & P B-C^{T}(\alpha I-D) \\
B^{T} P-(\alpha I-D)^{T} C & D^{T} D-\alpha\left(D+D^{T}\right)+a b I
\end{array}\right] \leq 0 .
$$

The proof of this theorem is presented in the Appendix. Note that, for $a=-1$ and $b=1$, the conditions in (ii) are equivalent to the bounded reainess lemma $[4,5]$, and the linear matrix inequality in (iii) corresponds to an LMI condition for norm-bounded systems [6,11]. These state-space characterizations of sector-bounded systems allow the use of reliable numerical algorithms for solution of linear matrix inequalities and algebraic Riccati inequalities, to determine sector-bounds for a given LTI system from its state-space realizations. The existence of a symmetric, positive definite solution to the linear matrix inequality (LMI) of condition (iii) may be established using convex programming techniques as in [6]. Alternately, if $\hat{R}=D^{T} D-\alpha\left(D+D^{T}\right)+a b I<0$, the LMI can be equivalently expressed as the algebraic Riccati inequality

$$
\begin{array}{r}
A^{T} P+P A-\left[P B-C^{T}(\alpha I-D)\right] \tilde{R}^{-1} \\
{\left[P B-C^{T}(\alpha I-D)\right]^{T}+C^{T} C \leq 0,}
\end{array}
$$

using a Schur complements identity. Positive definite solutions of this algebraic Riccati inequality may be obtained from a solution of the corresponding algebraic Riccati equation and using the results on comparative solutions of these equations [11, 12].

For linear, time-invariant systems, the notion of systems strictly inside a given sector in [1,2] is more restrictive than necessary to establish certain stability results. Following the approach in [4,5] for strictly bounded real systems and for strictly positive real systems [7], we propose the following definition of an LTI system to be strictly inside sector $[a, b]$.

Definition: A stable LTI system, $G(s)$, is said to be strictly inside sector $[a, b]$ if $\operatorname{Herm}\left\{[G(j \omega)-a I]^{*}[G(j \omega)-b I]\right\}<0$, for all $|\omega|<\infty$.

This definition for systems strictly inside a sector is weaker than that in previous literanure [1, 2], which corresponds to $\operatorname{Herm}\left\{[G(j \omega)-a I]^{*}[G(j \omega)-b I] \leq-\epsilon l\right.$, for all $\omega \in \mathbf{R}$, with $\epsilon>0$. Note that, with $a=-1$ and $b=1$, the new definition of LII systems strictly inside sector $[-1,1]$ reduces to the condition for strict, bounded realness of the system, that is, $\|G(s)\|_{\infty}<1$, as in [4,5]; whereas, under the previous definition, LTI systems strictly inside sector $[-1,1]$ corresponds to LTI system satisfying $\|G(s)\|_{\infty} \leq 1-\varepsilon$, for $\varepsilon>0$. For example, a transfer function, $\frac{s+1}{d+2}$, is strictly inside sector $[-1,1]$ with the new definition, but is not so under the previous definition. Theorems 3 and 4 show that the new (weaker) definition for systems satisfying sector conditions in a strict sense is adequate for stability results described therein. The following theorem characterizes LTI systems strictly inside sector $[a, b]$ in terms of its state-space realization, and is proved in the Appendix.

Theorem 2: A stable LTI system $\Sigma: \quad \dot{x}=A x+B u, y=C x+$ $D u$, where the quadruple $[A, B, C, D]$ is a minimal realization of the transfer function matrix $G(s)=C(s I-A)^{-1} B+D$, is strictly inside sector $[a, b]$ if and only if there exist real matrices $P=P^{T}>0, L$ and $W$ which satisfy

$$
\begin{aligned}
& P A+A^{T} P=-C^{T} C-L^{T} L, \\
& P B=C^{T}(\alpha I-D)-L^{T} W \\
& a b I-\alpha\left(D+D^{T}\right)+D^{T} D=-W^{T} W
\end{aligned}
$$

where $\alpha=(a+b) / 2$, and that the quadruple $[A, B, L, W]$ is a minimal realization of the transfer function $V(s)=$ $L(s I-A)^{-1} B+W$, which does not have transmission zeros on the imaginary axis (that is, rank $[V(j \omega)]=m$, for all $|\omega|<\infty)$.

\section{Stability Results}

Negative feedback interconnection of LTI systems, as shown in Figure 3, are known to be stable if the systems satisfy certain sector conditions given in [1-3]. This result is repeated here to demonstrate that the weaker conditions to characterize systems strictly inside a given sector are adequate for stability of the closed-loop system, and to present a direct Lyapunov functionbased proof for these results.

Theorem 3: Consider two stable. LTI systems $\Sigma_{i}, i=1,2$, with minimal state-space realizations, $\dot{x}_{i}=A_{i} x_{i}+B_{i} u_{i}, y_{i}=$ $C_{i} x_{i}+D_{i} u_{i}$, interconnected in the standard negative feedback configuration, shown in Figure 3. If $\Sigma_{1}$ is inside sector $[a, b]$, with $b>0>a$, and $\Sigma_{2}$ is inside the sector $\left[-\frac{1}{b},-\frac{1}{a}\right]$, then the origin is a Lyapunov stable equilibrium point of the closed-loop system. If either $\Sigma_{1}$ or $\Sigma_{2}$ satisfies the sector conditions in a strict sense, then the origin is an asymptotically stable equilibrium point. 
A straightforward Lyapunov function based proof to Theorem 3 is presented in the Appendix. Note that substituting $a=-1$ and $b=1$ in Theorem 3 leads to a small gain theorem for stability of the feedback interconnection of LTI systems shown in Figure 3. The importance of this result stems from the fact that it can be used for less conservative robust controllers as opposed to those based on the small gain theorem. While characterizing uncertainty by the $\mathcal{H}_{\infty}$ norm of the uncertain plant, the frequency response of the plant must lie within a circle in the frequency plane, whose center must be at the origin. Tighter bounds for the uncertainty can be achieved by allowing the center of this circle to move along the real axis, as is provided by the sector boundedness condition. With tighter bounds on the uncertainty in terms of sector conditions, the result in Theorem 3 characterizes a larger set of compensators which provide robust stability, and consequently leads to synthesis of less conservative robust compensators. For example, by Theorem 3, stable closedloop system is achieved with feedback around the plant $G(s)$ in Figure 2, for all compensators, $H(s)$, whose frequency response is within the circle corresponding to sector $[-1.0,2.5]$. This circle is shown in Figure 4, along with a unit circle centered at the origin, within which the frequency response must lie to guarantee stability by the small gain theorem. Thus, sector conditions for stability characterize a larger set of compensators that provide closed-loop stability. Specifically. a compensator $H(s)=15 / s^{2}+4 s+8$, whose Nyquist plot is shown in Figure 4, would lead to a stable closed-loop system by Theorem 3; however, the small gain theorem cannot be used to establish closed-loop stability. Synthesis of compensators within a given sector $[a, b]$ can be performed using techniques suggested in [10].

Another stability result in sector theory considers the stability of linear systems with memoryless nonlinearities in the feedback loop [13,14]. Let a minimal state-space realization of a stable LTI system be $\Sigma: \quad \dot{x}=A x+B u, \quad y=C x+D u$, and let $\psi(y, t)$ be a memoryless nonlinearity, which may be time-varying. The problem is to obtain sufficient conditions for the stability of the negative feedback interconnection of the LTI system and the nonlinearity, as shown in Figure 5. Time-varying, nonlinear closed-loop equations of this system are $\dot{x}=A x-B \psi(y, t), \quad y=C x-D \psi(y, t)$. Theorem 4 below shows that under mild conditions on the nonlinearity, the new definition of LTI systems satisfying sector conditions in a strict sense is adequate to establish asymptotic stability of the closedloop system above.

Theorem 4: Consider the negative feedback interconnection of a stable LTI system $\Sigma: \quad \dot{x}=A x+B u, y=C x+D u$, where the quadruple $[A, B, C, D]$ is a minimal realization, and a memoryless, time-varying, nonlinearity, $\psi(y, t)$ (as shown in Figure 5). If the nonlinearity, $\psi(y, t)$, is locally Lipschitz in $y$, uniformly Lipschitz in $t$, and belongs to sector $[a, b]$, with $b>0>a$, then the origin is a Lyapunov stable equilibrium point of the closed-loop system if $\Sigma$ is inside sector $\left[-\frac{1}{b},-\frac{1}{a}\right]$. If $\Sigma$ is strictly inside sector $\left[-\frac{1}{b},-\frac{1}{a}\right]$, then the origin is a globally asymptotically stable equilibrium point of the closed-loop system.

The proof of this theorem is given in the Appendix. Note that the conditions on the nonlinearity $\psi(y, t)$ are not very restrictive and are needed for well-posed closed-loop system as well. Moreover, global asymptotic stability of the closed-loop is ensured when the nonlinearity satisfies the sector condition for all $y \in R^{m}$. However, if the nonlinearity satisfies the sector condition in some local neighborhood of the origin, then this theorem guarantees (local) asymptotic stability about the origin. Theorem 4 presents a direct approach to this problem rather than applying loop transformations to convert sector $[a, b]$ into sector $[0, \infty)$ nonlinearities $[11,12]$. Similar to Theorem 3 . weaker constraints for LTI systems satisfying sector conditions in a strict sense make these results more general than those presented previously in the literature.

The results presented in this paper consider LTI systems inside sector $[a, b]$ with $b>0>a$. Corresponding results for a larger class of sectors can be developed with a similar approach [15]. For example, the negative feedback interconnection of an LTI system, $\Sigma_{1}$, inside sector $[0, b]$, for $b>0$, with another LTI system, $\Sigma_{2}$, such that $\left(\Sigma_{2}+b I\right)$ is strictly positive real, is stable, as expected from [1,2].

\section{Conclusions}

A state-space characterization of stable linear time-invariant systems inside sector $[a, b]$ in terms of a sector boundedness lemma and linear matrix inequalities has been presented. Moreover, weaker conditions for LTI systems to be strictly inside a sector are introduced. These weaker conditions are shown to be adequate for establishing sector conditions for (1) stability of the negative feedback interconnection of LTI systems, as well as, (2) the stability of LTI systems with sectorbounded feedback nonlinearities. Furthermore, a straightforward proof for these results is presented using the Lyapunov function approach, in contrast to the operator-theoretic methods used in previous works. Finally, it is argued, using the interpretation of sector-boundedness of LTI systems in terms of circles in the frequency plane, that less conservative robust controllers may be synthesized with these results, as opposed to those based on the small gain theorem.

\section{References}

[1] Zames, G., "On the Input-Output Stability of TimeVarying Nonlinear Feedback Systems, Parts I \& 2," IEEE Transactions on Automatic Control, Volume AC-11, Number 2 \& 3, April \& July 1966, pages $228-238$ \& 465-476.

[2] Willems, J. C., The Analysis of Feedback Systems, MIT Press. Cambridge, MA, 1972.

[3] Safanov, M. G., Stability and Robustness of Multivariable Feedback Systems, MTT Press, Cambridge. MA, 1980.

[4] Anderson, B. D. O. and Vongpanitlerd. S., Network Analysis and Synthesis: A Modern Systems Theory Approach, PrenticeHall, Englewood Cliffs, NJ, 1973.

[5] Haddad, W. M. and Bemstein, D. S., "Explicit Construction of Quadratic Lyapunov Functions for the Small Gain, Positivity, Circle, and Popov Theorems and Their Application to Robust Stability. Part I Continuous Time Theory" International Journal of Robust and Nonlinear Control, Volume 3, pages 313-339, 1993.

[6] Boyd, S., El Ghaoui, L., Feron, E. and Balakrishnan, V., Linear Matrix Inequalities in System and Control Theory, SIAM, Philadelphia, PA, 1994.

[7] Lozano-Leal, R. and Joshi, S. M., "Strictly Positive Real Transfer Functions Revisited," IEEE Transactions on Automatic Control, Volume AC-35, Number 11, 1990, pages 1243-1245. 
[8] Bamett, S., Matrices in Control Theory, Robert E. Krieger Publishing Company, Malabar, FL, 1984.

[9] Youla, D. C., "On the Factorization of Rational Matrices," IRE Transactions on Information Theory. Volume IT-7, pages 172-189, 1961.

[10]Safanov, M. G., Jonckheere, E. A., Verma, M., and Limebeer, D. J. N., "Synthesis of Positive Real Multivariable Feedback Systems," International Journal of Control, Volume 45, No. 3. pages 817-842, 1987.

[11]Zhou, K., Doyle, J. C. and Glover, K., Robust and Optimal Control, Version 94.7, July 1994.

[12]Ran, A. C. M. and Vreugdenhil, R., "Existence and Comparison Theorems for Algebraic Riccati Equations for Continuous- and Discrete-Time Systems," Linear Algebra and Its Applicarions, Volume 99, pages 63-83, 1988.

[13]M. Vidyasagar, Nonlinear System Analysis, Prentice-Hall, Englewood Cliffs, New Jersey, Second Edition, 1992.

[14]Khalil, H. K., Nonlinear Systems, Macmillian Publishing Company, New York, 1992.

[15]Gupta, S., "State-space Characterization and Robust Stabilization of Dissipative Systems," PhD Dissertation, The George Washington University, Washington D. C., May 1994.

\section{Appendix}

\section{Proof of Theorem 1:}

(i) $\Rightarrow$ (ii): Note that $\phi(s) \triangleq G^{\sim}(s) G(s)-\alpha\left[G(s)+G^{\sim}(s)\right]+$ $a b I$, where $\alpha=(a+b) / 2$, is parahermitian. If the linear system is inside sector $[a, b]$, then, by definition, $\phi(j \omega) \leq 0$, for all $\omega \in \mathbf{R}$. From the spectral factorization theorem [8, 9], there exists a stable transfer function, $V(s)$, with no transmission zeros in the open right-half plane, such that

$$
\phi(s)=-V^{\sim}(s) V(s) \text {. }
$$

If the quadruple $\left[A_{1}, B_{1}, C_{1}, D_{1}\right]$ is a minimal realization of $V(s)$, then $\left[-A_{1}^{T},-C_{1}^{T}, B_{1}^{T}, D_{1}^{T}\right]$ is a minimal realization for $V^{\sim}(s)$, and a minimal realization for the product $V^{\sim}(s) V(s)$ is given by

$$
\left[\left(\begin{array}{cc}
A_{1} & 0 \\
-C_{1}^{T} C_{1} & -A_{1}^{T}
\end{array}\right),\left(\begin{array}{c}
B_{1} \\
-C_{1}^{T} D_{1}
\end{array}\right),\left(\begin{array}{ll}
D_{1}^{T} C_{1} & B_{1}^{T}
\end{array}\right), D_{1}^{T} D_{1}\right] .
$$

Let $X_{1}=X_{1}^{T}>0$ be the observability grammian, that is, $X_{1}$ satisfies the Lyapunov equation

$$
A_{1}^{T} X_{1}+X_{1} A_{1}+C_{1}^{T} C_{1}=0 .
$$

Applying the state transformation $\left[\begin{array}{cc}I & 0 \\ X_{1} & I\end{array}\right]$, another minimal realization for the product $V^{\sim}(s) V(s)$ is given by

$$
\begin{aligned}
& {\left[\left(\begin{array}{cc}
A_{1} & 0 \\
0 & -A_{1}^{T}
\end{array}\right),\left(\begin{array}{c}
B_{1} \\
-X_{1} B_{1}-C_{1}^{T} D_{1}
\end{array}\right),\right.} \\
& \left.\left(B_{1}^{T} X_{1}+D_{1}^{T} C_{1} \quad B_{1}^{T}\right), D_{1}^{T} D_{1}\right] \text {. }
\end{aligned}
$$

Repeating this process with $G(s)$, which has a minimal realization $[A, B, C, D]$, a minimal realization of the product $G^{\sim}(s) G(s)$, is given by

$$
\begin{aligned}
& {\left[\left(\begin{array}{cc}
A & 0 \\
0 & -A^{T}
\end{array}\right),\left(\begin{array}{c}
B \\
-X B-C^{T} D
\end{array}\right),\right.} \\
& \left.\left(B^{T} X+D^{T} C \quad B^{T}\right), D^{T} D\right],
\end{aligned}
$$

where $X=X^{T}>0$ is the symmetric positive definite observability grammian of $G(s)$, that is, $X$ satisfies

$$
A^{T} X+X A+C^{T} C=0 .
$$

Therefore, $\phi(s)$ has a minimal realization

$$
\begin{aligned}
& {\left[\left(\begin{array}{cc}
A & 0 \\
0 & -A^{T}
\end{array}\right),\left(\begin{array}{c}
B \\
-X B+C^{T}(\alpha I-D)
\end{array}\right),\right.} \\
& \left(B^{T} X-(\alpha I-D)^{T} C \quad B^{T}\right) \text {, } \\
& \left.D^{T} D-\alpha\left(D+D^{T}\right)+a b \Gamma\right]
\end{aligned}
$$

From (1), (3) and (5), we conclude (as in [14]) that there exists a nonsingular matrix $T$ such that

$$
\begin{aligned}
& A_{1}=T^{-1} A T ; \quad B_{1}=T^{-1} B \\
& B_{1}^{T} X_{1}+D_{1}^{T} C_{1}=-\left[B^{T} X-(\alpha I-D)^{T} C\right] T \\
& D_{1}^{T} D_{1}=-\left[D^{T} D-\alpha\left(D+D^{T}\right)+a b I\right]
\end{aligned}
$$

Premultiplying (2) by $T^{-T}$ and postmultiplying by $T^{-1}$ results in

$$
\begin{gathered}
\left(T^{-T} A_{1}^{T} T^{T}\right) T^{-T} X_{1} T^{-T}+T^{-T} X_{1} T^{-1}\left(T A_{1} T^{-1}\right) \\
=-T^{-T} C_{1}^{T} C_{1} T^{-1} .
\end{gathered}
$$

Adding (4) and (7) leads to $P A+A^{T} P=-C^{T} C-L^{T} L$, where $P=X+T^{-T} X_{1} T^{-1}, P=P^{T}>\overline{0}$, and $L=C_{1} T^{-1}$. Serting $D=W_{1}$ and rearranging the terms in (6) leads to the other two relations in (ii).

(i) $\Leftarrow$ (ii): Substituting $G(j \omega)=C(j \omega I-A)^{-1} B+D$, in $\phi(j \omega)=G^{*}(j \omega) G(j \omega)-\alpha\left[G(j \omega)+G^{*}(j \omega)\right]+a b I$ lesds to

$$
\begin{aligned}
\phi(j \omega)=B^{T} & \left(-j \omega I-A^{T}\right)^{-1} C^{T} C(j \omega I-A)^{-1} B+ \\
& B^{T}\left(-j \omega I-A^{T}\right)^{-1} C^{T}(D-\alpha I)+ \\
& (D-\alpha I)^{T} C(j \omega I-A)^{-1} B+ \\
& D^{T} D-\alpha\left(D+D^{T}\right)+a b I .
\end{aligned}
$$

Using the matrix relations in (ii) leads to

$$
\begin{gathered}
\phi(j \omega)=-B^{T}\left(-j \omega I-A^{T}\right)^{-1} L^{T} L(j \omega I-A)^{-1} B \\
-B^{T}\left(-j \omega I-A^{T}\right)^{-1}\left[P A+A^{T} P\right](j \omega I-A)^{-1} B \\
-B^{T}\left(-j \omega I-A^{T}\right)^{-1}\left[P B+L^{T} W\right] \\
-\left[B^{T} P+W^{T} L\right] C(j \omega I-A)^{-1} B+W^{T} W .
\end{gathered}
$$

Using the identity

$$
\begin{gathered}
B^{T}\left(-j \omega I-A^{T}\right)^{-1}\left(P A+A^{T} P\right)(j \omega I-A)^{-1} B= \\
-B^{T} P(j \omega I-A)^{-1} B-B^{T}\left(-j \omega I-A^{T}\right)^{-1} P B
\end{gathered}
$$

and some algebraic manipulations lead to

$$
\begin{aligned}
\phi(j \omega)= & -B^{T}\left(-j \omega I-A^{T}\right)^{-1} L^{T} L(j \omega I-A)^{-1} B \\
& -B^{T}\left(-j \omega I-A^{T}\right)^{-1} L^{T} W \\
& -W^{T} L(j \omega I-A)^{-1} B-W^{T} W \\
= & -V^{*}(j \omega) V(j \omega) \leq 0
\end{aligned}
$$


for all $\omega \in \mathbf{R}$, where $V(j \omega)=L(j \omega I-A)^{-1} B+W$.

(ii) $\Leftrightarrow$ (iii): This equivalence follows directly by noting that

$$
\begin{gathered}
{\left[\begin{array}{cc}
C^{T} C+P A+A^{T} P & P B-C^{T}(\alpha I-D) \\
B^{T} P-(\alpha I-D)^{T} C & D^{T} D-\alpha\left(D+D^{T}\right)+a b I
\end{array}\right]} \\
=-\left[\begin{array}{cc}
L^{T} L & L^{T} W \\
W^{T} L & W^{T} W
\end{array}\right] \leq 0 .
\end{gathered}
$$

\section{Proof of Theorem 2:}

If $\phi(j \omega)<0$, for all $|\omega|<\infty$, then $\phi(j \omega)=$ $-V^{*}(j \omega) V(j \omega)$, where $V(s)=L(s I-A)^{-1} B+W$, as in the proof of Theorem 1. Further, since $\phi(j \omega)$ is negative definite on the imaginary axis, $V(j \omega)$ is full rank on the imaginary axis. that is, it does not have transmission zeros on the imaginary axis. Conversely, the proof of Theorem 1 shows that the sectorboundedness lemma leads to $\phi(j \omega)=-V^{*}(j \omega) V(j \omega)$. Since $V(s)$ is full rank on the imaginary axis, $\phi(j \omega)<0$, for all $|\omega|<\infty$. Further, it is always possible to select $L$ such that transmission zeros of $V(s)$ are in the open left-half plane $[8,9]$.

\section{Proof of Theorem 3:}

Stability of the closed-loop system is demonstrated using the following Lyapunov function $V\left(x_{1}, x_{2}\right)=V_{1}\left(x_{1}\right)-a b V_{2}\left(x_{2}\right)$, where $V_{i}\left(x_{i}\right)=x_{1}^{T} P_{i} x_{i}$, with $P_{i}=P_{i}^{T}>0$ being a positive definite matrix which satisfies the sector-boundedness lemma, for $i=1,2$. Note that since $b>0>a, V\left(x_{1}, x_{2}\right)$ is a positive definite function of $x_{1}$ and $x_{2}$. Time derivative of $V\left(x_{1}, x_{2}\right)$ is $\dot{V}\left(x_{1}, x_{2}\right)=\dot{V}_{1}\left(x_{1}\right)-a b \dot{V}_{2}\left(x_{2}\right)$. By Theorem 1. for $i=1,2$, if $\Sigma_{i}$ is inside sector $\left[a_{i}, b_{i}\right]$, then there exist matrices $P_{i}=P_{i}^{T}>0, L_{i}$ and $W_{i}$, which satisfy

$$
\begin{aligned}
& P_{i} A_{i}+A_{i}^{T} P_{i}=-C_{i}^{T} C_{i}-L_{i}^{T} L_{i}, \\
& P_{i} B_{i}=C_{i}^{T}\left(\alpha_{i} I-D_{i}\right)-L_{i}^{T} W_{i}, \\
& D_{i}^{T} D_{i}-\alpha_{i}\left(D_{i}+D_{i}^{T}\right)+a_{i} b_{i} I=-W_{i}^{T} W_{i},
\end{aligned}
$$

where $\alpha_{i}=\left(a_{i}+b_{i}\right) / 2$. Consider $\dot{V}_{i}\left(x_{i}\right)=\dot{x}_{i}^{T} P_{i} x_{i}+x_{i}^{T} P_{i} \dot{x}_{i}$ $=x_{i}^{T}\left(A_{i}^{T} P_{i}+P_{i} A_{i}\right) x_{i}+u_{i}^{T} B_{i}^{T} P_{i} x_{i}+x_{i}^{T} P_{i} B_{i} u_{i}$. Using the first two relations in $(8)$ leads to

$$
\begin{aligned}
\dot{V}_{i}\left(x_{i}\right)= & -x_{i}^{T} L_{i}^{T} L_{i} x_{i}-x_{i}^{T} L_{i}^{T} W_{i} u_{i}-u_{i}^{T} W_{i}^{T} L_{i} x_{i} \\
& -x_{i}^{T} C_{i}^{T} C_{i} x_{i}-x_{i}^{T} C_{i}^{T} D_{i} u_{i}-u_{i}^{T} D_{i}^{T} C_{i} x_{i} \\
& +\alpha_{i} x_{i}^{T} C_{i}^{T} u_{i}+\alpha_{i} u_{i}^{T} C_{i} x_{i}
\end{aligned}
$$

Adding and subtracting $u_{i}^{T} W_{i}^{T} W_{i} u_{i}$ for "completing the square," using the last relationship in (8) and some algebraic manipulations, results in

$$
\begin{gathered}
\dot{V}_{i}\left(x_{i}\right)=-\left[L_{i} x_{i}+W_{i} u_{i}\right]^{T}\left[L_{i} x_{i}+W_{i} u_{i}\right] \\
-\left(y_{i}-b_{i} u_{i}\right)^{T}\left(y_{i}-a_{i} u_{i}\right)
\end{gathered}
$$

With these expressions for $\dot{V}_{1}\left(x_{1}\right)$ and $\dot{V}_{2}\left(x_{2}\right)$, the time derivative of the Lyapunov function $V\left(x_{1}, x_{2}\right)$ becomes

$$
\begin{aligned}
\dot{V}\left(x_{1}, x_{2}\right)= & -\left(L_{1} x_{1}+W_{1} u_{1}\right)^{T}\left(L_{1} x_{1}+W_{1} u_{1}\right) \\
& -\left(y_{1}-b u_{1}\right)^{T}\left(y_{1}-a u_{1}\right) \\
& +a b\left(L_{2} x_{2}+W_{2} u_{2}\right)^{T}\left(L_{2} x_{2}+W_{2} u_{2}\right) \\
& +a b\left(y_{2}+u_{2} / a\right)^{T}\left(y_{2}+u_{2} / b\right)
\end{aligned}
$$

Using $u_{2}=y_{1}$ and $u_{1}=-y_{2}$ as implied by the negative feedback interconnection, $a b\left(y_{2}+u_{2} / a\right)^{T}\left(y_{2}+u_{2} / b\right)=$ $\left(y_{1}-b u_{1}\right)^{T}\left(y_{1}-a u_{1}\right)$. Therefore,

$$
\begin{aligned}
\dot{V}\left(x_{1}, x_{2}\right)= & -\left(L_{1} x_{1}+W_{1} u_{1}\right)^{*}\left(L_{1} x_{1}+W_{1} u_{1}\right) \\
& -(-a b)\left(L_{2} x_{2}+W_{2} u_{2}\right)^{*}\left(L_{2} x_{2}+W_{2} u_{2}\right) \\
\leq & 0 .
\end{aligned}
$$

Thus, the origin is a Lyapunov stable equilibrium of the closedloop system.

When one of the system satisfies the sector conditions in a strict sense, asymptotic stability of the closed-loop is proved using LaSalle's Invariant Set Theorem $[13,14]$. Suppose $\Sigma_{2}$ is strictly inside sector $\left[-\frac{1}{b},-\frac{1}{a}\right]$. We demonstrate, by contradiction, that $x_{1}=x_{2}=0$ is the only trajectory for which $\dot{V}\left(x_{1}, x_{2}\right)=0$. If $\dot{V}\left(x_{1}, x_{2}\right)=0$, then (9) implies that $y_{2}^{\prime}=L_{2} x_{2}+W_{2} u_{2}=0$. If $u_{2} \neq 0$, then it has to be an exponentially decreasing function of time. since transmission zeros of $V_{2}(s)$ are in the open left half plane. Stability of the linear systems and the minimality of their realizations imply that $x_{1}$ and $x_{2}$ are exponentially decreasing functions of ime, leading to $\dot{V}\left(x_{1}, x_{2}\right)<0$, which is a contradiction. Thus, the origin is an asymptotically stable equilibrium point of the closed-loop system. A similar argument holds if $\Sigma_{1}$ satisfies the sector conditions in a strict sense.

\section{Proof of Theorem 4:}

Consider a Lyapunov function, $V(x)=x^{T} P x$, where $P=P^{T}>0$ is a symmetric positive definite matrix which satisfies the sector-boundedness lemma in Theorem 1. From the proof of Theorem 3, we get

$$
\dot{V}(x)=-[L x+W u]^{T}[L x+W u]-(y+u / a)^{T}(y+u / b)
$$

Since the nonlinearity, $\psi(y, t)$, is inside sector $[a, b]$. $(\psi-b y)^{T}(\psi-a y) \leq 0$, for all $y$. The negative feedback condition implies that $u=-\psi$. Thus, we have $(u+b y)^{T}(u+a y) \leq 0$, or, $(y+u / a)^{T}(y+u / b) \geq 0$. Thus, from (10) we have $\dot{V}(x) \leq 0$, and the origin is Lyapunov stable.

If $\Sigma$ satisfies the sector conditions in a strict sense, and $\dot{V}(x)=0$, then $x=0$ is the only possible trajectory of the system, by a contradiction argument as in Theorem 3 . Thus, by the Invariance Theorems in [14], the closed-loop system is asymptotically stable. 


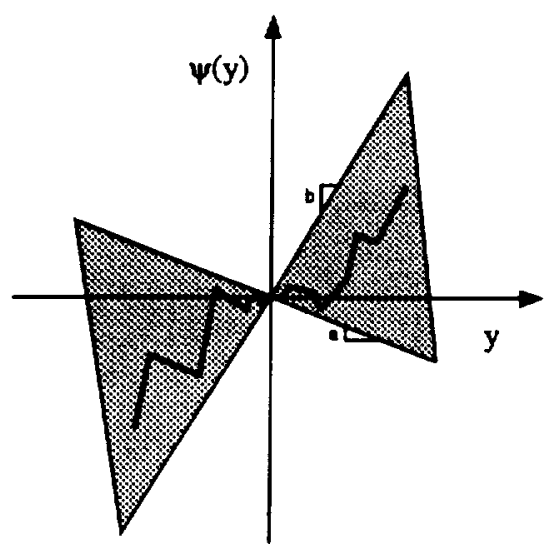

Figure 1. Memoryless Nonlinearity Inside Sector [a,b].

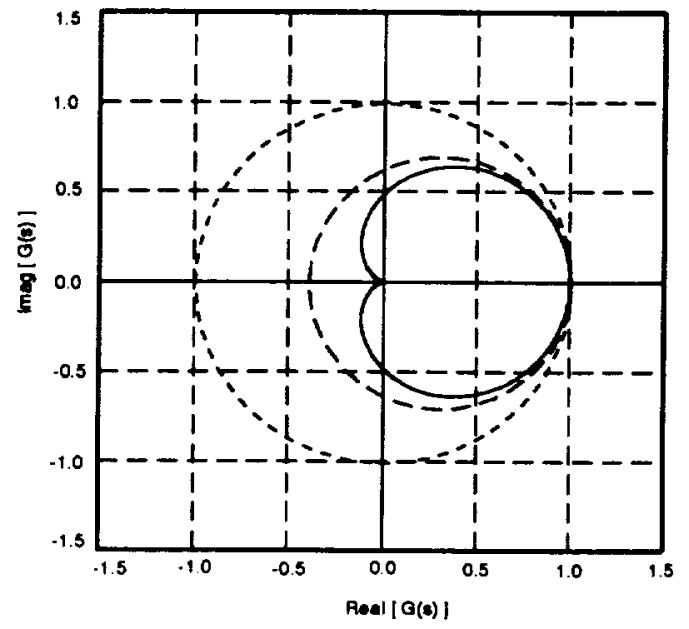

Figure 2. Nyquist Plot of $G(s)=15 /(s+3)(s+5)$ Inside Sector $[-0.4,1.0]$.

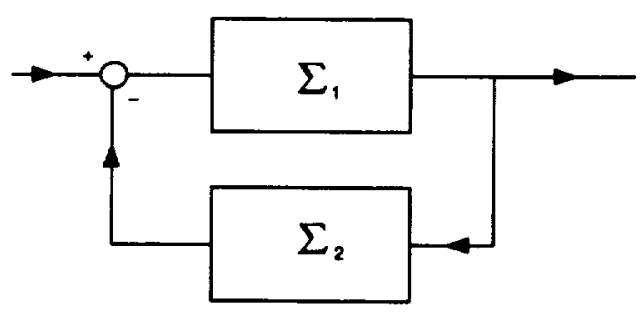

Figure 3. Negative feedback interconnection of two LTI systems, $\Sigma_{1}$ and $\Sigma_{2}$.

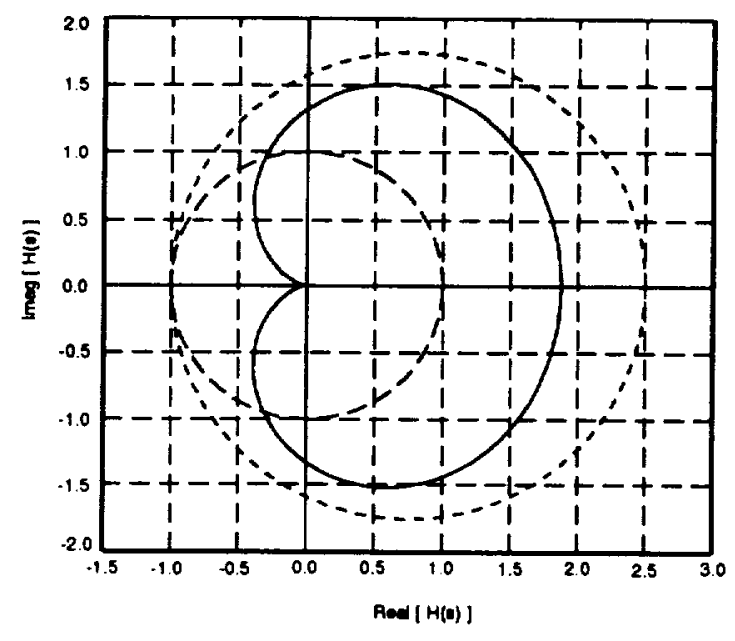

Figure 4. Nyquist Plot of $H(s)=15 /\left(s^{\wedge} 2+4 s+8\right)$ Inside Sector $[-1.0,2.5]$.

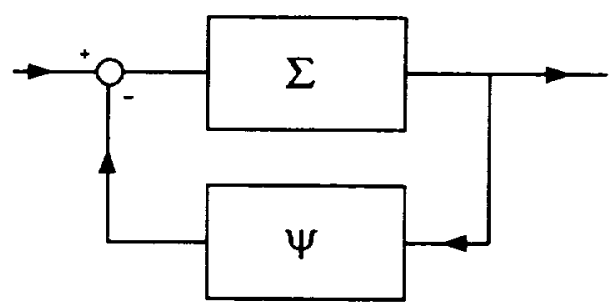

Figure 5. Absolute stability framework, with nonlinearity, $\psi$, in negative feedback about an LTI system, $\Sigma$. 
